\title{
Physiological and molecular responses of two Chinese cabbage genotypes to heat stress
}

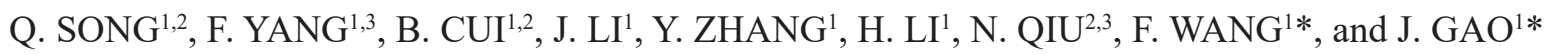 \\ Institute of Vegetables and Flowers, Shandong Academy of Agricultural Sciences; Shandong Key Laboratory \\ of Greenhouse Vegetable Biology and Shandong Branch of National Vegetable Improvement Center, Jinan, \\ 250100, P.R. China ${ }^{1}$ \\ College of Life Sciences, Shandong Normal University, Jinan, 250014, P.R. China ${ }^{2}$ \\ College of Life Sciences, Qufu Normal University, Qufu, 273165, P.R. China ${ }^{3}$
}

\begin{abstract}
A comparative investigation of heat stress-mediated physiological and biochemical parameters in conjunction with the expression analysis of heat shock transcription factors (BrHSF) from two different Chinese cabbage genotypes was done to understand the mechanism of heat tolerance. Our results show that the heat-tolerant (2013-33) genotype had a smaller relative electric conductivity, a less malondialdehyde content and a higher maximal efficiency of photosystem II photochemistry than the heat-sensitive (AM160) genotype, and was able to develop the leaf head under heat stress, whereas 'AM160' flailed to develop it. The results also indicate that '2013-33' accumulated a higher amount of soluble sugars and protein under heat stress condition than 'AM160'. However, it warrants to mention that proline content and antioxidant enzymes, such as the peroxidase, catalase, and superoxide dismutase activities, in the 2013-33 genotype under HS were recorded, being significantly lower than in 'AM160'. Additionally, the expression profile of BrHSF genes was checked and classified to three main groups, (i) HS-induced HSFs expressed in both genotypes ( group I), (ii) suppressed by HS in both genotypes (groupII), and (iii) genotype-specific expression of HSFs (repressed in the AM160 heat-sensitive genotype whereas induced in the '2013-33' heat tolerance genotype; group III). Furthermore, the result of promoter analysis shows that group III BrHSFs, i.e., 23, 30, and 33 gene promoter regions possessed a difference between '2013-33' and 'AM160'. In conclusion, the results of our study identify that '2013-33' had more heat tolerance than 'AM160' because of a higher accumulation of sugar and protein and an enhanced expression of group III HSFs, and the differential response of group III $H S F S$ to HS in these two genotypes may be because of a promoter sequence difference. The study provides us a clue towards understanding the mechanism of heat tolerance in Chinese cabbage and offers a valuable source for further improvement of heat tolerance in Chinese cabbage.
\end{abstract}

Additional key words: antioxidants, chlorophyll fluorescence, heat shock, malondialdehyde, proline, proteins, sugars, transcription factor.

\section{Introduction}

Heat stress is one of the environmental cues limiting crop growth and productivity worldwide (Bita and Gerats 2013, Hasanuzzaman et al. 2013, Long and Ort 2010). Cropbased model analysis has indicated that a $1^{\circ} \mathrm{C}$ increase in seasonal temperature leads to $2.5-16 \%$ direct yield losses for essential crops in tropical and subtropical regions (Lobell et al. 2008). Due to enhancing anthropogenic activities following the increasing concentration of the greenhouse gasses, the global mean temperature was predicted to rise by $0.3{ }^{\circ} \mathrm{C}$ per decade, which seriously threatens the food security (Jones et al. 1999, Wahid et al. 2007).

Submitted 15 October 2018, last revision 29 December 2018, accepted 2 January 2019.

Abbreviations: CAT - catalase; $\mathrm{F}_{\mathrm{v}} / \mathrm{F}_{\mathrm{m}}$ - variable to maximum chlorophyll fluorescence ratio; HSC - heat stress conditions; HSF - heat shock transcriptional factor; MDA - malondialdehyde; NC - normal conditions; POD - peroxidase; PS - photosystem; REC - relative electric conductivity; ROS - reactive oxygen species; SNP - single nucleotide polymorphism; SOD - superoxide dismutase.

Acknowledgments: This work was supported by the National Natural Science Foundation of China (31471884); the National Key Research and Development Program of China (2017YFD0101801); the Modern Agricultural Industrial Technology System Funding of Shandong Province, China (SDAIT-05-04); the Prospect of Shandong Seed Project, China (2016LZGC033); the China Agriculture Research System (CARS-25); and the Key Research and Development Program of Shandong Province, China (2018GNC110011, 2018GNC110003). The first two authors contributed equally to this paper.

* Corresponding authors e-mails: wfengde@163.com; jianweigao3@yahoo.com 
Heat stress causes significant impacts on various physiological and metabolic processes in plants, such as membrane injury, protein inactivation, reactive oxygen species (ROS) production and metabolic destruction (Quinn 1988, Iba 2002,). As sessile organisms, plants have evolved various physiological and molecular mechanisms to resist heat stress such as accumulation of compatible osmolytes, an increase of heat shock proteins, production of antioxidants, as well as synthesis of secondary metabolites (Wahid et al. 2007, Hasanuzzaman et al. 2013). During this process, a heat shock transcriptional factor (HSF), which widely exists in plants to regulate the expression of heat shock proteins and other stress proteins, plays a central role in modulating heat stress response (Baniwal et al. 2004, Kotak et al. 2007, Qu et al. 2013, Scharf et al. 2012, Xue et al. 2015).

The HSFs contain a highly conserved N-terminal DNA-binding domain, which recognizes special elements (5'-AGAAnnTTCT-3') in promoters of heat stress-inducible genes (Nover et al. 2001, Scharf et al. 2012). Based on the structural features of their oligomeric domains, plant HSF proteins can be sorted into three classes: $A, B$, and $C$ (Nover et al. 2001), and class $A$ HSFs contains the AHA motif that is essential for transcriptional activation, whereas classes $B$ and $C$ HSFs do not possess this motif (Kotak et al. 2004). Among them, class $A$ HSFs has been relatively well studied, and the results show that HSFA1a is a master regulator and HsfA2 is a major heat stress factor in plant heat stress responses (Scharf et al. 2012). Up to now, the HSF family has been thoroughly identified in various plants such as Arabidopsis (Nover et al. 2001), tomato (Yang et al. 2016), soybean (Li et al. 2014), potato (Tang et al. 2016), maize (Lin et al. 2011), as well as Chinese cabbage (Song et al. 2014) with a fully sequenced genome.

Chinese cabbage (Brassica rapa L. ssp. pekinensis) is one of the most important leafy vegetables in Asia, and the leaf head is the standard edible part. In the heading stage, $15^{\circ} \mathrm{C}$ to $18{ }^{\circ} \mathrm{C}$ is the adaptable growth temperature for Chinese cabbage, and a mean temperature higher than $25^{\circ} \mathrm{C}$ usually causes difficulty in forming leaf head (Ke 2010). Therefore, the production of Chinese cabbage is severely threatened by heat stress in many regions. At present, physiological parameters of Chinese cabbage regarding heat stress response have been identified (Yang et al. 2017). Unfortunately, to our knowledge, a systematic analysis of physiological, biochemical, and heat stressinduced HSF family genes in responses to heat stress in Chinese cabbage is ignored, especially under the natural growth conditions.

In this study, physiological responses, such as the maximal efficiency of photosystem (PS) II photochemistry $\left(\mathrm{F}_{\mathrm{v}} / \mathrm{F}_{\mathrm{m}}\right)$, biochemical parameters, such as soluble sugar content, antioxidant enzymes, and other parameters were measured in two genotypes of Chinese cabbage with different heat tolerance potentials under normal (August 14 to October 14, Jinan, China) and heat stress (June 1 to August 1, Jinan, China) growth conditions. Additionally, the real time quantitative PCR based expression profiles of the HSF family genes were detected under the same growth conditions. The objectives of this study were: (1) physiological responses of Chinese cabbage under heat stress conditions; (2) the role of HSF genes in heat-stress tolerance in Chinese cabbage; and (3) to promote future breeding strategies directed towards enhancing heat stress tolerance in Chinese cabbage.

\section{Materials and methods}

Plant materials and growth conditions: The inbred lines AM160 and 2013-33 of Chinese cabbage (Brassica rapa L. ssp. pekinensis), which are heat sensitive and heat tolerant genotypes, respectively, were chosen for this study. The seeds from these two inbred lines were sowed in the field at the Vegetable and Flower Research Institute, the Shandong Academy of Agricultural Sciences farm station (Jinan, China) from June 1 to August 1 heat stress conditions (HSC) and from August 14 to October 14 normal conditions (NC) in 2017, respectively. Plants at the end of rosette stage with 8-10 expanded leaves were marked for sampling. The same position full extend leaves from individual plants were harvested and immediately frozen in liquid nitrogen and stored at $-80^{\circ} \mathrm{C}$ for subsequent physiological parameter measurements and RNA isolation.

Determination of PSII photochemistry and relative electric conductivity: The maximal efficiency of PSII photochemistry $\left(\mathrm{F}_{\mathrm{v}} / \mathrm{F}_{\mathrm{m}}\right)$ was determined using a plant efficiency analyzer (Handy PEA, Hansatech Instrument $L t d$., UK) by measuring a modulated light at a constant light intensity of $3000 \mu \mathrm{mol} \cdot \mathrm{m}^{-2} \cdot \mathrm{s}^{-1}$.

Leaf relative electric conductance (REC) was determined according to the method described by Wang et al. (2011). The REC was evaluated as REC [\%] = $=(\mathrm{S} 1 / \mathrm{S} 2) \times 100$, where $\mathrm{S} 1$ and $\mathrm{S} 2$ refer to the electric conductivity of live leaves and boiled leaves, respectively.

Determination of malondialdehyde, proline, soluble sugars and soluble protein content: Leaf malondialdehyde (MDA) content was determined according to the method of Hodges et al. (1999). In brief, a total of $1.0 \mathrm{~g}$ of fresh leaves was homogenized in $10 \mathrm{~cm}^{3}$ of $10 \%(\mathrm{~m} / \mathrm{v})$ trichloroacetic acid. After centrifugation at $12000 \mathrm{~g}$ for $6 \mathrm{~min}, 2 \mathrm{~cm}^{3}$ of the supernatant and $2 \mathrm{~cm}^{3}$ $0.6 \%(\mathrm{~m} / \mathrm{v})$ thiobarbituric acid was mixed and boiled for 15 minutes. Absorbances were measured at 450, 532 and $600 \mathrm{~nm}$ after cooling down to room temperature.

Leaf proline content was determined using the method of Bates et al. (1973). Proline was extracted from $1.0 \mathrm{~g}$ of fresh samples into $10 \mathrm{~cm}^{3}$ of $3 \%(\mathrm{~m} / \mathrm{v})$ sulfosalicylic acid and by using the L-proline as a standard; absorbance was taken at $520 \mathrm{~nm}$.

The Jermyn (1975) method was used for leaf soluble sugar content measurement. Firstly, a total of $1.0 \mathrm{~g}$ of fresh leaves was homogenized in $10 \mathrm{~cm}^{3}$ of water. After centrifugation at $12000 \mathrm{~g}$ for $6 \mathrm{~min}$, a $1 \mathrm{~cm}^{3}$ of the supernatant was added to $0.5 \mathrm{~cm}^{3}$ of $2 \%(\mathrm{~m} / \mathrm{v})$ anthrone and $5 \mathrm{~cm}^{3} 98 \%(\mathrm{~m} / \mathrm{m}) \mathrm{H}_{2} \mathrm{SO}_{4}$. When the mixture was cooled down to room temperature, absorbance was recorded at 
$620 \mathrm{~nm}$ using glucose as a standard. Leaf soluble protein content was determined according to the method of Bradford (1976) using bovine serum albumin as a protein concentration standard.

Determination of antioxidant enzyme activities: Totally $1.0 \mathrm{~g}$ of fresh leaves was homogenized in $10 \mathrm{~cm}^{3}$ of a cold buffer containing potassium phosphate $(50 \mathrm{mM}$, pH 7.0), $20 \%$ (v/v) glycerol, $1 \mathrm{mM}$ DTT, $1 \mathrm{mM} \mathrm{Na}{ }_{2}$ EDTA, $1 \%$ PVP $(\mathrm{m} / \mathrm{v})$, and $5 \mathrm{mM} \mathrm{MgSO}$. After centrifugation at $12000 \mathrm{~g}$ for $6 \mathrm{~min}$ and immediately then at $26000 \mathrm{~g}$ for $15 \mathrm{~min}$, the supernatant was transferred to a new tube for subsequent peroxidase (POD), catalase (CAT), and superoxide dismutase (SOD) measurements.

Leaf POD activity was determined according to the method described by Chance and Maehly (1955) by monitoring the absorbance of tetraguaiacol at $470 \mathrm{~nm}$. A reaction mixture contained potassium phosphate $(50 \mathrm{mM}$, $\mathrm{pH} 7.0), 5 \mathrm{mM} \mathrm{H}_{2} \mathrm{O}_{2}, 18 \mathrm{mM}$ guaiacol and $0.02 \mathrm{~cm}^{3}$ of the supernatant.

Leaf CAT activity was determined according to the method described by Knöraer et al. (1996) by monitoring the absorbance of $\mathrm{H}_{2} \mathrm{O}_{2}$ at $240 \mathrm{~nm}$. A reaction mixture contained potassium phosphate $(50 \mathrm{mM}, \mathrm{pH} 7.0), 10 \mathrm{mM}$ $\mathrm{H}_{2} \mathrm{O}_{2}$, and $0.1 \mathrm{~cm}^{3}$ of the supernatant.

Leaf SOD activity was determined according to the method described by Beyer and Fridovich (1987). A reaction mixture contained potassium phosphate $(50 \mathrm{mM}$, $\mathrm{pH} 7.5), 13 \mathrm{mM}$ methionine, $75 \mu \mathrm{M}$ nitro blue tetrazolium, $10 \mu \mathrm{M} \mathrm{Na} \mathrm{NaDTA}_{2}, 2 \mu \mathrm{M}$ riboflavin and $0.02 \mathrm{~cm}^{3}$ of the supernatant. After the reaction, absorbance was recorded at $560 \mathrm{~nm}$, and one unit of SOD was defined as the amount of the extract needed for a $50 \%$ decrease in nitro blue tetrazolium reduction.

Real-time quantitative PCR: For real time quantitative PCR, the total RNA was first extracted from the leaves of the two genotypes of Chinese cabbage under NC (June 1 to August 1 2017) and HSC (August 14 to October 14 2017) using TRNzol $A^{+}$(Tiangen, Beijing, China). Then, first strand cDNA was synthesized from $1 \mu \mathrm{g}$ of the total RNA using a PrimeScript $R T$ reagent kit with $g D N A$ Eraser (Takara, Dalian, China). Real time quantitative PCR was carried out using an SYBR Green PCR master mix (Takara) on a CFX96 Optics real-time PCR system (Bio-Rad, Hercules, CA, USA). In this experiment, actin was used as a constitutive expression control. Real time quantitative PCR was performed according to a previous report (Qiu et al. 2017). The relative expression level was calculated by the comparative $2^{-\Delta \mathrm{Ct}}$ method.

Promoter analysis of the BrHSFs: For cloning promoter regions of $B r H S F 9,21,23,30$, and 33 genes, the sequences of $1500 \mathrm{bp}$ upstream to the start codon of these genes were taken for designing specific primers. Then, PCR amplified promoter DNA fragments were cloned into the pMD18-T cloning vector (Takara) and sequenced using an $A B I$ 3730XL DNA Sequencer. The identification of cis-acting regulatory elements (CARE) was carried out on an online software (PlantCARE, http://bioinformatics.psb.ugent.be/ webtools/plantcare/html/).

\section{Results and discussion}

Physiological influence of heat stress on Chinese cabbage: A series of cellular injuries and even cell death have been reported under acute heat stress due to membrane injury, protein inactivation, production of ROS, and metabolic destruction (Quinn 1988, Iba 2002,). These injuries lead to an inhibition of growth, productivity, and even plant death (Wahid et al. 2007). Repression of the frequency of leaf head formation and physiological injury at a higher mean temperature $\left(>25^{\circ} \mathrm{C}\right)$ has been reported in Chinese cabbage (Ke 2010). Therefore, two different heat tolerance genotypes of Chinese cabbage were selected for the study, and the seeds were sown in the field (Farm station, Jinan, China) twice in a year (i.e., from June 1 to August 1 and from August 14 to October 14 2017). According to our recording, a mean temperature from June 1 to August 1 was $27.8{ }^{\circ} \mathrm{C}$ (Fig. 1 Suppl.). Especially at the heading stage (July 12 to August 1) a mean temperature was even as high as $28.7^{\circ} \mathrm{C}$, and only the 2013-33 genotype usually formed the leaf head (Fig. 2 Suppl.). Even if from September 10 to October 14 (the heading stage), a mean temperature was only $20.9{ }^{\circ} \mathrm{C}$ (Fig. 1 Suppl.), both the genotypes customarily formed the leaf head (Fig. 2 Suppl.). The results suggest that the temperature from June 1 to August 1 had a significant influence on heading in Chinese cabbage and can be defined as heat stress conditions (HSC), whereas the temperature from August 14 to October 14 can be described as normal conditions (NC) for the selected genotypes since it has no noticeable influence on them. Additionally, the maximal efficiency of PSII photochemistry $\left(\mathrm{F}_{\mathrm{v}} / \mathrm{F}_{\mathrm{m}}\right)$ had no significant difference on '2013-33' and 'AM160' under $\mathrm{NC}$, whereas $\mathrm{F}_{\mathrm{v}} / \mathrm{F}_{\mathrm{m}}$ in 'AM160' was significantly lower than in '2013-33' under HSC (Fig. 1A). Therefore, the result of $\mathrm{F}_{\mathrm{v}} / \mathrm{F}_{\mathrm{m}}$ reconfirmed that the period June - August imposed a significant influence on Chinese cabbage heading and could be defined as HSC.

Abiotic stresses, such as heat, drought, and salt, usually cause ROS production and membrane lipid peroxides, leading to the increase of membrane permeability and electrolyte leakage rate (Quinn 1988, Iba 2002, Cramer et al. 2011). Malondialdehyde content and REC are important traits and could present the level of membrane lipid peroxides and membrane permeability, respectively (Asthir 2015). An increased membrane permeability and electrolyte leakage rate in Chinese cabbage have been identified under heat stress (Luo et al. 1996, Ye et al. 1996). Therefore, we also determined the effect of heat stress on electrolyte leakage in the two genotypes. An increase of REC was recorded in the 2013-33 and AM160 genotypes under both NC and HSC. However, the REC in '2013-33' was substantially lesser than in 'AM160' (Fig. 1B). The result of our study is consistent with the research of electrolyte leakage in Chinese cabbage (Luo et al. 1996). Additionally, MDA 


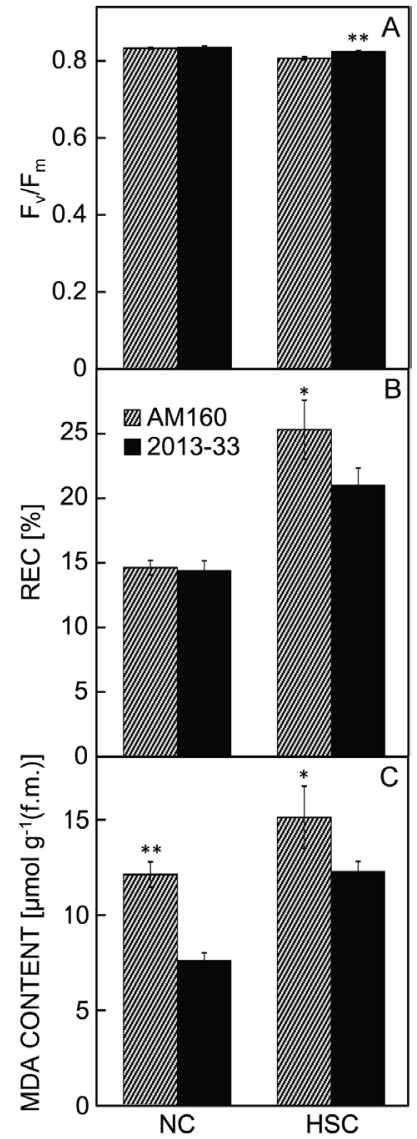

Fig. 1. The influence of heat stress on Chinese cabbage 'AM160' and '2013-33': $A$ - maximum efficiency of photosystem II photochemistry $\left(\mathrm{F}_{\mathrm{v}} / \mathrm{F}_{\mathrm{m}}\right) ; B$ - relative electric conductivity (REC); $C$ - content of malondialdehyde (MDA). Means \pm SDs, $n=10$, 3 , and 3 for $\mathrm{F}_{\mathrm{v}} / \mathrm{F}_{\mathrm{m}}, \mathrm{REC}$, and MDA, respectively; * and ** significant differences at $P<0.05$ and $P<0.01$. NC - normal conditions, HSC - heat stress conditions.

content was also checked in the two genotypes, and the result shows that an accumulation of MDA was identified in '2013-33' and 'AM160' under NC and HSC (Fig. 1C). It is worthwhile to mention that under NC and HSC, MDA content in '2013-33' was still lower than in 'AM160' (Fig. 1C). Our results are in line with the study of Wang et al. (2005) on Chinese cabbage. The results of $\mathrm{F}_{\mathrm{v}} / \mathrm{F}_{\mathrm{m}}$, REC, and MDA content show that these parameters have a tight correlation with heat tolerance and could be used as good physiological traits for the identification of heat tolerance genotypes of Chinese cabbage and other crop plants as well.

Biochemical responses of Chinese cabbage to exposure to heat stress: Plants have evolved various strategies to reduce the damage induced by heat stress (Wahid et al. 2007). The methods that are universally employed by plants to enhance heat tolerance and to cope with heat stress as well include accumulation of compatible osmolytes, an improved expression of heat shock proteins, production of antioxidants, and secondary metabolite syntheses (Wahid et al. 2007, Hasanuzzaman et al. 2013).

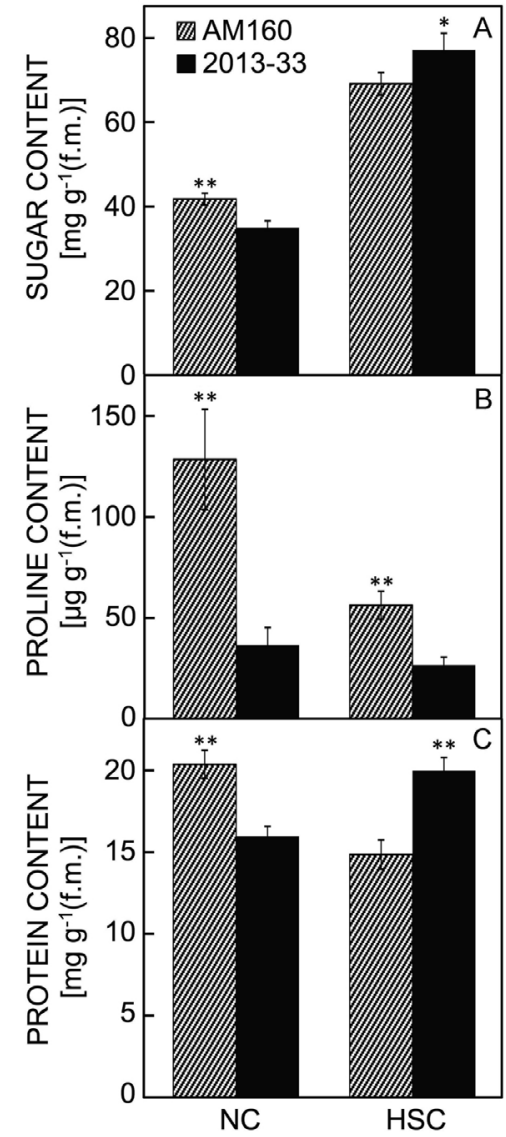

Fig. 2. The heat stress responses in Chinese cabbage 'AM160' and '2013-33': $A$ - content of soluble sugars; $B$ - content of proline; $C$ - content of soluble proteins. Means \pm SDs, $n=3$; $*$ and ** - significant differences at $P<0.05$ and $P<0.01$. NC normal conditions, HSC - heat stress conditions.

Accumulation of soluble sugars and proline have been identified as compatible osmolytes, which significantly contributes to an enhanced plant heat stress tolerance (Wahid 2007, Wahid and Close 2007). Accumulation of soluble sugars was associated with improved heat and drought stress tolerance of the sid2-1 mutant of Arabidopsis (Kumazaki and Suzuki 2018). Here, the high temperature induced accumulation of soluble sugars in both the genotypes, higher in '2013-33' than in 'AM160' under HSC (Fig. 2A). However, a reduced proline content was recorded in both the genotypes under HSC (Fig. 2B). This result confirms the previous findings of plant-specific proline accumulation in response to HSC (Bokszczanin et al. 2013). For example, proline content significantly increases in heat stressed leaves of tomato (Rivero et al. 2004) and tobacco (Cvikrová et al. 2012), whereas it only slightly accumulated in heat stressed leaves of chickpea (Chakraborty and Tongden 2005), barley and radish (Chu et al. 1974). Even more, proline content does not alter in heat stressed Arabidopsis (Hua et al. 2001, Rizhsky et al. 2004 , Yoshiba et al. 1995), and is even reduced in heat stressed germinating wheat seeds (Song et al. 2014). Interestingly, the high temperature induced enhanced proteins content in '2013-33' compared to 'AM160' (Fig. 2C). Similar results 


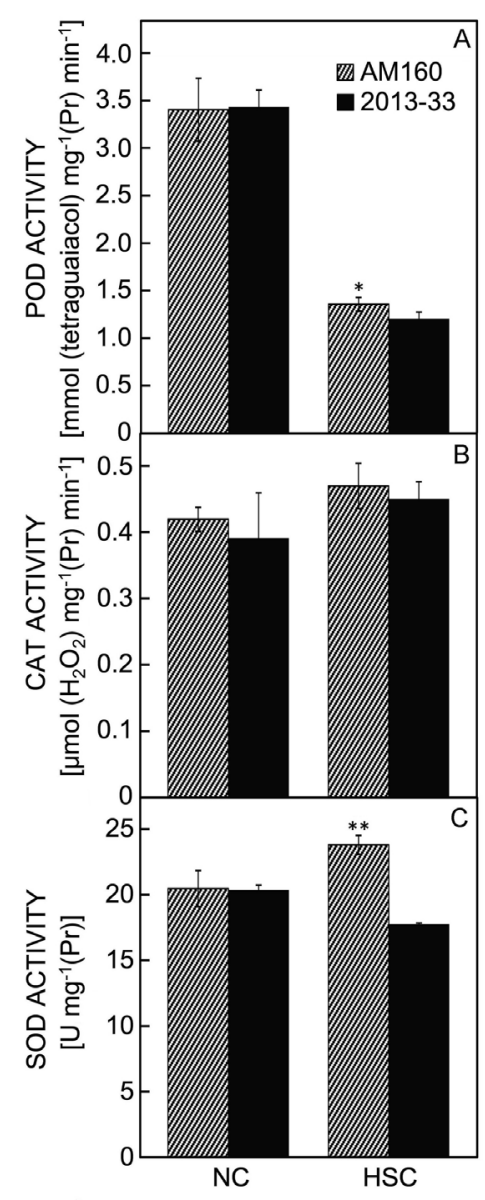

Fig. 3. The activities of antioxidant enzymes in Chinese cabbage 'AM160' and '2013-33' under normal (NC) and heat stress (HSC) conditions: $A$ - peroxidase (POD); $B$ - catalase (CAT); $C$ - superoxide dismutase (SOD). Means \pm SDs, $n=3$; * and ** - significant differences at $P<0.05$ and $P<0.01$. NC - normal conditions, HSC - heat stress conditions.

were also reported in Chinese cabbage (Wang et al. 2005). The HSC induced accumulation of a heat shock protein (Wahid et al. 2007, Hasanuzzaman et al. 2013), ubiquitin (Sun and Callis 1997), and late embryogenesis abundant protein (Arora et al. 1998, Wahid and Close 2007), have been reported to protectcellular and sub-cellular structures against oxidative and dehydration forces (Ortiz and Cardemil 2001, Li et al. 2014, Mishra et al. 2018,). The results of enhanced accumulation of proteins in the heat tolerant genotype 2013-33 may be attributed to its heat stress tolerance.

Accumulated ROS under HSC causes autocatalytic peroxidation of membrane lipids thus leading to the loss of membrane stability (Wahid et al. 2007, Hasanuzzaman et al. 2013). Peroxidase, CAT, and SOD are three antioxidant enzymes, which have been identified to play crucial roles to cope with ROS produced under HSC (Wahid et al. 2007). Therefore, we have measured their activities in the two genotypes of Chinese cabbage. In this study, POD activity was found to be reduced in both the genotypes, and even it significantly declined in '2013-33' (Fig. $3 A$ ). Although enhanced CAT activities were recorded in both the genotypes under HSC, no significant difference in CAT activity between '2013-33' and 'AM160' under $\mathrm{NC}$ and $\mathrm{HSC}$ were found $(3 B)$. Being congruous with POD and CAT activities, the high temperature inhibited SOD activity in '2013-33', whereas it was enhanced in the AM160 genotype (Fig. 3C). Our findings are entirely different from a previous study of Ye et al. (1996) with an improved SOD, CAT, and POD activities in a heat tolerant cultivar (Yashu No. 1) under heat stress, whereas activities of SOD and POD decreased in a heat-sensitive variety (106), though with a slightly increased CAT activity. However, Wu et al. (1995) reported a declining POD activity, whereas CAT activity increases during heat treatment, and POD activity in a heat-tolerant genotype was higher than in a heat-sensitive genotype in both control and heat treatments.

Expression profiling Chinese cabbage HSF genes in response to heat stress: Reports have indicated that HSFs play a central role in regulation of the expressions of HSPs and other stress-responsive proteins under heat stress (Baniwal et al. 2004, Kotak et al. 2007, Scharf et al. 2012, Qu et al. 2013). Thirty-five $H S F$ genes have been identified in Chinese cabbage and were classified to $A, B$, and $C$ groups (Song et al. 2014). Therefore, in this study, to understand the function of $B r H S F$ genes in heat tolerance in Chinese cabbage, the $35 \mathrm{BrHSF}$ genes were subjected to real-time quantitative PCR based expression profiling across the 2013-33 and AM160 genotypes under $\mathrm{NC}$ and HSC. Based on the expression profiles, a heat map was generated, and clustering analysis was done by using a multiple experiment viewer (Fig. 4). The clustering analysis

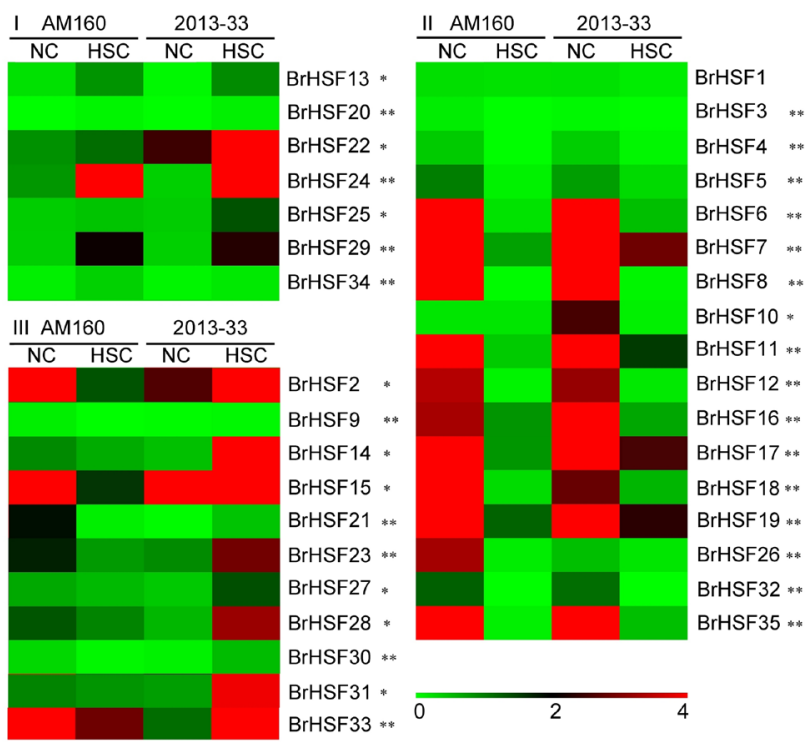

Fig. 4. The expression of BrHSF genes in Chinese cabbage 'AM160' and '2013-33' under normal (NC) and heat stress (HSC) conditions. I - induced by heat stress in both; II - suppressed by heat stress in both; III - suppressed in AM160, induced in '201333 '. * - the expression varied more than two times just in one genotype, ${ }^{* *}$ - the expression varied more than two times in both the genotypes. 
classified the BrHSFs into three groups viz: induced by heat stress in both the genotypes (group I), suppressed by heat stress in both the genotypes (group II), and repressed in the heat-sensitive genotype whereas induced in the heat tolerance genotype (group III, Fig. 4). However, it warrants mentioning that the results of our study contrast with the previous research of Song et al. (2014), in which all BrHSF genes of Chinese cabbage were up-regulated under a heat treatment. The possible reason for different results could be due to the use of a controlled environment growth chamber for the treatment, and the five-leaf stage chosen for the treatment (Song et al. 2014). In this study, the plants were grown under a natural environment, i.e., in the field, and were continuously subjected to the long-term heat stress (Fig. 1 Suppl.).

A tomato HSFAla gene has been identified as a master regulator for acquired thermo-tolerance (Mishra et al. 2002). However, no AtHSFA1 gene has a comparable role with the master regulator since knocking-out every single HSFAl gene as well as knocking-out double or triple HSFA1 genes have no significant defects in the overall heat stress response of Arabidopsis (Lohmann et al. 2004, Nishizawa-Yokoi et al. 2011). But, knockingout four AtHSFA1 genes, the mutant plant shows a severely impaired heat stress (HS) response, and this suggests these four AtHSFA1 genes together as a "master regulator" (Liu et al. 2011). Eight BrHSFA1 genes have been identified in Chinese cabbage: Nos. 2, 5, 6, 12, 21, 26, 29, and 32, and were shown to have a similar expression profile (Song et al. 2014). However, the results of our study show that the genes of the BrHSFA1 group of transcription factors did not have the same expression profiles as shown in Fig. 4. The BrHSF29 was categorized into group I, and BrHSF5, 6, 12, 26, and 32 belonged to group II, whereas BrHSF2 and BrHSF21 were classified into group III (Fig. 4). Hence, the expression profile-based analysis unveiled diversity in functions of BrHSFAl genes in response to the heat stress. However, a possibility that the gene of BrHSFAl transcription factors may act as a "master regulator" of heat stress response in Chinese cabbage remains elusive. However, our study has provided a platform for understanding the roles of BrHSF genes in heat stress response.

Previously, the classes $B$ and $C$ HSFs were usually identified with no transcriptional activity because of the absence of AHA motif (Scharf et al. 1990, CzarneckaVerner et al. 2000, Li et al. 2014). However, a recent research evidenced that they play essential roles in the modulation of heat stress response in plants (Ikeda et al. 2011, Zhang et al. 2013). Furthermore, HSFB1 and HSFB2b positively regulate an acquired thermotolerance in Arabidopsis thaliana (Ikeda et al. 2011). Additionally, the over-expression of an HSFB2 subgroup gene (TaHSF3) of wheat significantly increases heat tolerance in transgenic Arabidopsis (Zhang et al. 2013). Ten and two heat stress transcription factors $(H S F s)$ of class $B$ and class $\mathrm{C} B r H S F s$, respectively, were characterized in Chinese cabbage (Song et al. 2014). Here, the analysis of the expression profile shows that two class $B$ BrHSF genes (BrHSF22 and 25) belonged to group I, that six class
B BrHSF genes (BrHSF1, 7, 8, 11, 18, and 35) fitted to group II, and that two class $B$ BrHSF (BrHSF9 and 31) and two class $C B r H S F$ genes (BrHSF14 and 23) belonged to group III (Fig. 4). A Similarresult was also found in the previous studies on Chinese cabbage (Song et al. 2014) as well as on other plants in which class $B$ and class $C$ HSFs genes were induced by heat stress (Nover et al. 2001, Tang et al. 2016, Yang et al. 2016).

The differences of $B r H S F$ promoter sequences between '2013-33' and 'AM160': Further, to understand the differential mechanisms of the heat stress expression profile of group III BrHSFs $(2,9,14,15,21,23,27,28$, 30,31 , and 33) across the two genotypes, $\operatorname{BrHSFs}(9,21$, 23,30 , and 33) with $>2$-fold changes in their expression profile were subjected to promoter analysis. Promoter regions ( $\sim 1500$ bp up-stream to the start codon) for BrHSFs $9,21,23,30$, and 33 genes from the two genotypes were sequenced and subjected to PlantCARE database analysis for promoter analysis. The result of promoter analysis shows that the promoter regions of only three $B r H S F s$, i.e. 23, 30, and 33, possessed a difference between '201333 ' and 'AM160'. Among them, BrHSF23, BrHSF30, and $B r H S F 33$ promoter regions contained 30 single nucleotide polymorphisms (SNPs) and 2 Indels, 2 SNPs and 1 Indel, and 18 SNPs and one Indel, respectively (Fig. 1 Suppl.). Additionally, the CARE database was checked, and it was found that some CARE caused by a sequence variation disappeared or were formed in the promoter region (Table 1 Suppl.). These results suggest the different response of these two genotypes to the heat stress maybe due to the promoter sequence difference because some of the changed CARE have a tight correlation with the response to various stresses. For example, ABRE is the cognate cis-element for ABRE-binding factors, which is a crucial transcription factor mediating abscisic acid signaling and abscisic acidregulated stress response (Yoshida et al. 2010, Yoshida et al. 2015, Wang et al. 2018). Additionally, the G-box is a cognate cis-element for bZIP, bHLH, and NAC proteins, and the $\mathrm{W}$ box is a cognate cis-element for WRKY proteins (Liu et al. 2016). Of course, there are other factors also involved in the different responses to the heat stress in these two genotypes since no sequence difference was found in $\mathrm{BrHSF} 9$ and 21 promoter regions between 'AM160' and '2013-33'. In any case, it gives us a good clue to understanding the mechanism of different responses to heat stress of $B r H S F$ genes although it is a very complicated regulating process.

\section{Conclusions}

To our knowledge, this study first systematically analyzes the impact of heat stress on Chinese cabbage regarding the physiological defense response, biochemical variables, and $H S F$ expression profiles. Firstly, the results show that the parameters $\mathrm{F}_{\mathrm{v}} / \mathrm{F}_{\mathrm{m}}, \mathrm{REC}$, and MDA content had a tight correlation with heat tolerance and could be used as good physiological traits for heat tolerance genotype determination in Chinese cabbage. Secondly, accumulation of soluble sugars and soluble proteins maybe 
partly contribute to the heat tolerance in Chinese cabbage. Thirdly, the analysis of BrHSF expression profiles of heat stress response gave us a good clue to understanding its function and improving heat resistance in Chinese cabbage. Overall, the results of this study will be useful for us to build understanding the mechanism of heat tolerance in Chinese cabbage and provides a basis for creating Chinese cabbage cultivars with enhanced heat stress tolerance.

\section{References}

Arora, R., Pitchay, D.S., Bearce, B.C.: Water stress induced heat tolerance in geranium leaf tissues: A possible linkage through stress proteins? - Physiol. Plant. 103: 24-34, 1998.

Asthir, B.: Mechanisms of heat tolerance in crop plants. - Biol. Plant. 59: 620-628, 2015.

Baniwal, S.K., Bharti, K., Chan, K.Y., Fauth, M., Ganguli, A., Kotak, S., Mishra, S.K., Nover, L., Port, M., Scharf, K.D., Tripp, J., Weber, C., Zielinski, D., von Koskull-Döring, P.: Heat stress response in plants: a complex game with chaperones and more than twenty heat stress transcription factors. -J. Biosci. 29: 471-487, 2004.

Bates, L.S., Waldren, R.P., Teare, I.D.: Rapid determination of free proline for water stress studies. - Plant Soil 39: 205-207, 1973.

Beyer, W.F., Fridovich, I.: Assaying for superoxide dismutase activity: some large consequences of minor changes in condition. - Anal. Biochem. 161: 559-566, 1987.

Bita, C.E., Gerats, T.: Plant tolerance to high temperature in a changing environment: Scientific fundamentals and production of heat stress-tolerant crops. Front. Plant Sci. 4: 273, 2013.

Bokszczanin, K.L., Solanaceae Pollen Thermotolerance Initial Training Network (SPOT-ITN) Consortium, Fragkostefanakis, S.: Perspectives on deciphering mechanisms underlying plant heat stress response and thermotolerance. - Front. Plant Sci. 4: 315, 2013.

Bradford, M.M.: A rapid and sensitive method for the quantization of microgram quantities of protein utilizing the principle of protein-dye binding. - Anal. Biochem. 72: 248-254, 1976.

Chakraborty, U., Tongden, C.: Evaluation of heat acclimation and salicylic acid treatments as potent inducers of thermotolerance in Cicer arietinum L. - Curr. Sci. 89: 384-389, 2005.

Chance, B., Maehly, A.C.: Assay of catalase and peroxidases. In: Maehly, A.C. (ed.): Methods in enzymology. - Vol. 2 Pp. 764775. Acadamic Press, New York 1955.

Chu, T.M., Aspinall, D., Paleg, L.G.: Stress metabolism. VI. Temperature stress and the accumulation of proline in barley and radish. - Aust. J. Plant Physiol. 1: 87-97, 1974.

Cramer, G.R., Urano, K., Delrot, S., Pezzotti, M., Shinozaki, K.: Effects of abiotic stress on plants: a system biology perspective. - BMC Plant Biol. 11: 163, 2011.

Cvikrová, M., Gemperlová, L., Dobrá, J., Martincová, O., Prásil, I.T., Gubis, J., Vanková, R.: Effect of heat stress on polyamine metabolism in proline-overproducing tobacco plants. - Plant Sci. 182: 49-58, 2012.

Czarnecka-Verner, E., Yuan, C.X., Scharf, K.D., Englich, G., Gurley, W.B.: Plants contain a novel multi-member class of heat shock factors without transcriptional activator potential. - Plant Mol. Biol. 43: 459-471, 2000.

Hasanuzzaman, M., Nahar, K., Alam, M.M., Roychowdhury, R., Fujita, M.: Physiological, biochemical, and molecular mechanisms of heat stress tolerance in plants. - Int. J. Mol. Sci. 14: 9643-9684, 2013.
Hodges, D.M., DeLong, J.M., Forney, C.F., Prange R.K.: Improving the thiobarbituric acid-reactive-substances assay for estimating lipid peroxidation in plant tissues containing anthocyanin and other interfering compounds. - Planta 207: 604-611, 1999.

Hua, X.J., Van De Cotte, B., Van Montagu, M., Verbruggen, N.: The 5' untranslated region of the At-P5R gene is involved in both transcriptional and post-transcriptional regulation. Plant J. 26: 157-169, 2001.

Iba, K.: Acclimative response to temperature stress in higher plants: Approaches of gene engineering for temperature tolerance. - Annu. Rev. Plant Biol. 53: 225-245, 2002.

Ikeda, M., Mitsuda, N., Ohme-Takagi, M.: Arabidopsis HsfB1 and $\mathrm{HsfB} 2 \mathrm{~b}$ act as repressors of the expression of heatinducible Hsfs but positively regulate the acquired thermo tolerance. - Plant Physiol. 157: 1243-1254, 2011.

Jermyn, M.A.: Increasing the sensitivity of the anthrone method for carbohydrate. - Anal. Biochem. 68: 332-335, 1975.

Jones, P.D., New, M., Parker, D.E., Martin, S., Rigor, I.G.: Surface air temperature and its changes over the past 150 years. - Rev. Geophys. 37: 173-199, 1999.

Ke, G.: [Chinese Cabbage Breeding.] - China Agricul. Press, Beijing 2010. [In Chin.]

Knöraer, O.C., Durner, J., Böger, P.: Alterations in the antioxidative system of suspension-cultured soybean cells (Glycine max) induced by oxidatice stress. - Physiol. Plant. 97: 388-396, 1996.

Kotak, S., Port, M., Ganguli, A., Bicker, F., von Koskull-Doring, P.: Characterization of C-terminal domains of Arabidopsis heat stress transcription factors (Hsfs) and identification of a new signature combination of plant class A Hsfs with AHA and NES motifs essential for activator function and intracellular localization. - Plant J. 39: 98-112, 2004.

Kotak, S., Vierling, E., Bäumlein, H., von Koskull-Döring P.: A novel transcriptional cascade regulating expression of heat stress proteins during seed development of Arabidopsis. Plant Cell 19: 182-195, 2007.

Kumazaki, A., Suzuki, N.: Enhanced tolerance to a combination of heat stress and drought in Arabidopsis plants deficient in ICS1 is associated with modulation of photosynthetic reaction center proteins. - Physiol. Plant. 165: 232-246, 2019.

Li, H., Liu, S.S., Yi, C.Y., Wang, F., Zhou, J., Xia, X.J., Shi, K., Zhou, Y.H., Yu JQ.: Hydrogen peroxide mediates abscisic acid-induced HSP70 accumulation and heat tolerance in grafted cucumber plants. - Plant Cell Environ. 37: 2768-2780, 2014.

Li, P.S., Yu, T.F., He, G.H., Chen, M., Zhou, Y.B., Chai, S.C., Xu, Z.S., Ma, Y.Z.: Genome-wide analysis of the Hsf family in soybean and functional identification of $\mathrm{GmHsf}-34$ involvement in drought and heat stresses. - BMC Genomics 15: 1009, 2014.

Lin, Y.X., Jiang, H.Y., Chu, Z.X., Tang, X.L., Zhu, S.W., Cheng, B.J.: Genome-wide identification, classification and analysis of heat shock transcription factor family in maize. - BMC Genomics 12: 76, 2011.

Liu, H.C., Liao, H.T., Charng, Y.Y.: The role of class A1 heat shock factors (HSFA1s) in response to heat and other stresses in Arabidopsis. - Plant Cell Environ. 34: 738-751, 2011.

Liu, L., Xu, W., Hu, X., Liu, H., Lin, Y.: W-box and G-box elements play important roles in early senescence of rice flag leaf. - Sci. Rep. 6: 20881, 2016.

Lobell, D.B., Burke, M., Tebaldi, C., Mastrandrea, M.D., Falcon, W.P., Naylor, R.L.: Prioritizing climate change adaptation needs for food security in 2030. - Science 319: 607-610, 2008.

Lohmann, C., Eggers-Schumacher, G., Wunderlich, M., Schöffl, F.: Two different heat shock transcription factors regulate 
immediate early expression of stress genes in Arabidopsis. Mol. Genet. Genom. 271: 11-21, 2004.

Long, S.P., Ort, D.R.: More than taking the heat: Crops and global change. - Curr. Opin. Plant Biol. 13: 241-248, 2010.

Luo, S., Li, Z., Zhou, W., Keniehi, H., Takehiko, N.: [Technique for identification of heat tolerance in heading Chinese cabbage.] - China Vegetables 2: 16-18, 1996. [In Chin.]

Mishra, N., Srivastava, A.P., Esmaeili, N., Hu, W., Shen, G.: Overexpression of the rice gene OsSIZ1 in Arabidopsis improves drought-, heat-, and salt-tolerance simultaneously. - PLoS ONE 13: e0201716, 2018.

Mishra, S.K., Tripp, J., Winkelhaus, S., Tschiersch, B., Theres, K., Nover, L., Scharf, K.D.: In the complex family of heat stress transcription factors, HsfA1 has a unique role as master regulator of thermotolerance in tomato. - Genes Dev. 16:1555$1567,2002$.

Nishizawa-Yokoi, A., Nosaka, R., Hayashi, H., Tainaka, H., Maruta, T., Tamoi, M., Ikeda, M., Ohme-Takagi, M., Yoshimura, K., Yabuta, Y., Shigeoka, S.: HsfA1d and HsfA1e involved in the transcriptional regulation of HsfA2 function as key regulators for the Hsf signaling network in response to environmental stress. - Plant Cell Physiol. 52: 933-945, 2011.

Nover, L., Bharti, K., Döring, P., Mishra, S.K., Ganguli, A., Scharf, K.D.: Arabidopsis and the heat stress transcription factor world: how many heat stress transcription factors do we need? - Cell Stress Chaperon. 6: 177-189, 2001.

Ortiz, C., Cardemil, L.: Heat-shock responses in two leguminous plants: a comparative study. - J. exp. Bot. 52: 1711-1719, 2001.

Qiu, N., Liu, Q., Li, J., Zhang, Y., Wang, F., Gao, J.: Physiological and transcriptomic responses of Chinese cabbage (Brassica rapa L. ssp. Pekinensis) to salt stress. - Int. J. mol. Sci. 18: 1953, 2017.

Qu, A.L., Ding, Y.F., Jiang, Q., Zhu, C.: Molecular mechanisms of the plant heat stress response. - Biochem. Biophys. Res. Commun. 432: 203-207, 2013.

Quinn, P.J.: Effects of temperature on cell membranes. - Symp. Soc. exp. Biol. 42: 237-258, 1988.

Rivero, R.M., Ruiz, J.M., Romero, L. M.: Importance of N source on heat stress tolerance due to the accumulation of proline and quaternary ammonium compounds in tomato plants. - Plant Biol. 6: 702-707, 2004.

Rizhsky, L., Liang, H. J., Shuman, J., Shulaev,V., Davletova, S., Mittler, R.: When defense pathways collide. The response of Arabidopsis to a combination of drought and heat stress. Plant Physiol. 134: 1683-1696, 2004.

Scharf, K.D., Berberich, T., Ebersberger, I., Nover, L.: The plant heat stress transcription factor (Hsf) family: Structure, function and evolution. - Biochim. Biophys. Acta 1819: 104119, 2012.

Scharf, K.D., Rose. S., Zott, W., Schöffl, F., Nover, L.: Three tomato genes code for heat stress transcription factors with a region of remarkable homology to the DNA-binding domain of the yeast HSF. - EMBO J. 9: 4495-4501, 1990.

Song, X., Liu, G., Duan, W., Liu, T., Huang, Z.,Ren, J., Li, Y., Hou, X.: Genomewide identifcation, classifcation and expression analysis of the heat shock transcription factor family in Chinese cabbage. - Mol. Genet. Genom. 289: 541551, 2014.

Sun, C.W., Callis, J.: Independent modulation of Arabidopsis thaliana polyubiquitin mRNAs in different organs of and inresponse to environmental changes. - Plant J. 11: 10171027, 1997.

Tang, R., Zhu, W., Song, X., Lin, X., Cai, J., Wang, M., Yang, Q.: Genome-wide identification and function analyses of heat shock transcription factors in potato. - Front. Plant Sci. 7: 490, 2016.

Wahid, A.: Physiological implications of metabolite biosynthesis for net assimilation and heat-stress tolerance of sugarcane (Saccharum officinarum) sprouts. - J. Plant Res. 120: 219228, 2007.

Wahid, A., Close, T.J.: Expression of dehydrins under heat stress and their relationship with water relations of sugarcane leaves. - Biol. Plant. 51: 104-109, 2007.

Wahid, A., Gelani, S., Ashraf, M., Foolad, M.R.: Heat tolerance in plants: An overview. - Environ. exp. Bot. 61: 199-223, 2007.

Wang, H.S., Yu, C., Zhu, Z.J., Yu, X.C.: Overexpression in tobacco of a tomato GMPase gene improves tolerance to both low and high temperature stress by enhancing antioxidation capacity. - Plant Cell Rep. 30: 1029-1040, 2011.

Wang, X., Guo, C., Peng, J., Li, C., Wan, F., Zhang, S., Zhou, Y., Yan, Y.,Qi, L.,Sun, K., Yang, S., Gong, Z., Li, J.: ABREBINDING FACTORS play a role in the feedback regulation of ABA signaling by mediating rapid $\mathrm{ABA}$ induction of $\mathrm{ABA}$ co-receptor genes. - New Phytol. 221: 341-355, 2019.

Wang, Z., Yu, L., Cao, D., Zhou, B., Yang, J.: [The effect to several physiological indexes by short-term high temperature in Chinese cabbage.] - Acta Agr. Boreali-occidental. Sinica 14: 82-85, 2005. [In Chin.]

Wu, G., Cao,W., Wang, Y., Jiang, Y., Zhang, L.: [Cell membrane thermostability, protective enzymes and heat tolerance in Chinese cabbage.] - Acta Hort. Sinica 22: 353-358, 1995. [In Chin.]

Xue, G.P., Drenth, J., McIntyre, C.L.: TaHsfA6f is a transcriptional activator that regulates a suite of heat stress protection genes in wheat (Triticum aestivum L.) including previously unknown Hsf targets. - J. exp. Bot. 66: 1025-1039, 2015.

Yang, F., Liu, H., Qiu, N., Wang, F., Gao, J.: [Advances in research of heat resistance mechanism of Chinese cabbage.] Shandong Agricul. Sci. 49: 149-153, 2017. [In Chin.]

Yang, X., Zhu, W., Zhang, H., Liu, N., Tian, S.: Heat shock factors in tomatoes: genome-wide identification, phylogenetic analysis and expression profiling under development and heat stress. - Peer J. 4: e1961, 2016.

Ye, C., Ke, Y., Chen, W.: [A study on the physiology of heat tolerance in Chinese cabbage II. Metabolism of water and proteins in leaves and heat tolerance.] - J. Fujian Agricul. Univ. 25: 490-493, 1996. [In Chin.]

Yoshida, T., Fujita, Y., Maruyama, K., Mogami, J., Todaka, D., Shinozaki, K., Yamaguchi-Shinozaki, K.: Four Arabidopsis AREB/ABFtranscriptionfactors function predominantly in gene expressiondownstream of SnRK2 kinases in abscisic acid signalling in response to osmotic stress. - Plant Cell Environ. 38: 35-49, 2015.

Yoshida, T., Fujita, Y., Sayama, H., Kidokoro, S., Maruyama, K., Mizoi, J., Shinozaki, K., Yamaguchi-Shinozaki, K.: AREB1, AREB2, and ABF3 are master transcription factors that cooperatively regulate ABRE-dependent ABA signaling involved in drought stress tolerance and require $\mathrm{ABA}$ for full activation. - Plant J. 61: 672-685, 2010.

Yoshiba, Y., Kiyosue, T., Katagiri, T., Ueda, H., Mizoguchi, T., Yamaguchi-Shinozaki, K., Wada, K., Harada, Y., Shinozaki, $\mathrm{K}$.: Correlation between the induction of a gene for $\Delta 1$ pyrroline-5-carboxylate synthetase and the accumulation of proline in Arabidopsis thaliana under osmotic stress. - Plant J. 7: 751-760, 1995.

Zhang, S., Xu, Z.S., Li, P., Yang, L., Wei, Y., Chen, M., Li, L., Zhang, G., Ma, Y.: Overexpression of TaHSF3 in transgenic Arabidopsis enhances tolerance to extreme temperatures. Plant mol. biol. Rep. 31: 688-697, 2013. 